\title{
Exposures, mutations and the history of causality
}

The article by Trichopoulos and colleagues (in this issue) raises some interesting problems concerning the complex relations between exposures, cancer aetiology and pathogenesis, and in particular the role of mutations in DNA. I believe that, to better understand the intertwining between causes and mechanisms, it is useful to recall some of the most influential interpretations of causality.

An old, deeply rooted tradition on causality is represented by the aristotelian point of view, based on the central idea of material and efficient cause. This tradition had its maximum development in the medicine of the early 19th century, when Bichat stated that a necropsy can shed more light on disease than hundreds of physical examinations of patients. This tradition is founded (a) on morbid anatomy, that is on the identification of characteristic lesions left in the dead body by the cause of disease, and (b), especially after Virchow's contribution, on the identification of the cell as the main actor in the pathogenetic process. Another extension of this traditional paradigm is microbiology, with its search for agents that could meet Henle-Koch's criteria, that is a single, necessary and sufficient cause. The cause is an agent that is always identifiable in the lesions, can be observed, it is absent in healthy subjects, and induces the disease by inoculation into animals.

To such a tradition, which dominated science in the 19th century, a reaction arises in the first years of the last century, aiming to substitute the concept of functional "law" to that of material cause. The most important expression of this tendency can be found in Mach's theory-who proposed some typical themes of Hume's philosophy while criticising a naive and material conception of causes. Also this conception finds an equivalent in medicine, in the school of Virchow's disciples. Students such as Martius and Hueppe, in fact, developed causal models of a multifactorial kind, which we find in the current probabilistic interpretations of causality. They insisted on the fact that we should get rid of a unilateral conception of causality, aiming to search for necessary causes. According to Mach's functionalist philosophy, Martius interpreted the "disposition" (internal cause) and the "stimulus" (external cause) as variables subject to a mathematical treatment. According to the even more radical school of "conditionalists", the word "cause" itself had to be completely abandoned and replaced by "determining condition": "all the conditions in a process are equally important", that is no hierarchy can be established".

A third recent stream is represented by the development of modal logics and particularly of "conditional counterfactuals". The basic idea is that of a cause as a "conditio sine qua non" for the occurrence of the effect; thus, the background is still that of the necessary cause. One of the most representative philosophers in this stream of thought is Mackie, who suggested the concept of cause as INUS (Insufficient Non-redundant component of an Unnecessary Sufficient Complex). ${ }^{2}$ In other words, Mackie claims that there are not separate causes, but causal complexes; each of the different complexes is sufficient to induce the effect, but none of them is itself necessary. Within such complexes, however, "non-redundant" (that is, necessary) components can be isolated, and these represent the true causes (they are necessary to make the complex sufficient). This approach is not very effective to describe inferences in epidemiology, if these consider the individual subject: lung cancer can arise in the single person even in the absence of cigarette smoking, which is not a necessary cause. Smoking can become necessary only in the context of a particular causal complex; however, in carcinogenesis we have not found such examples yet. The counterfactual reasoning, though, is applicable at the population level: without cigarette smoking there would not have been the longlasting epidemic of lung cancer that has characterised developed countries and is now spreading to developing countries.

The use of counterfactual conditionals is accompanied by a hypothetic-deductive style of reasoning, and to induction "by elimination". A typical example is represented by the famous description, attributable to Hempel, of the story of Ignatz Semmelweis. ${ }^{3}{ }^{4}$ Hempel describes Semmelweis' inferential procedures as an example of "trial and error" approach: after the early epidemiological observations, which suggested the transmission of "particles" from dead bodies, he started seeking for evidence that would falsify his theory, and for alternative explanations. For example, he ruled out that puerperal fever had been contracted before hospitalisation because of the women's living conditions. He also excluded, partly by the means of experiments, several other potential confounders or sources of bias.

Still another peculiar and recent concept of cause has been introduced by Wesley Salmon through the idea of the "propagation - or trasmission - of a mark over time": a process is causal if it is based on the transmission of a certain mark or characteristic in the course of time. We go back, in this way, to the 19th century idea of a "localisation" of disease, that is the cause is an alteration that can be identified in the tissue and is characteristic of a certain agent (for example the tubercle for tuberculosis). The cause is, therefore, a kind of signature that persists over time. This is an extremely common concept in the history of medicine, now widespread thanks to the developments of molecular medicine: the "cause of cancer", for example, is identifed with a genetic alteration such as a mutation in the $p 53$ gene, or, as in the case discussed by Trichopoulos and colleagues in this issue, in the ras oncogene. According to the theory of molecular "fingerprints", a carcinogenic chemical would be able to leave a characteristic signature in a specific cancer gene, and this fingerprint would be transmitted through generations of cells until it is observable within the tumour. This is at the moment still a theoretical hypothesis with some observational basis. ${ }^{6}$

In fact, what Porta et al have shown, and is discussed by Trichopoulos and colleagues, is that subjects with pancreatic cancer showed mutations in the ras gene more frequently if they were heavy coffee drinkers, ${ }^{7}$ suggesting a complex causal pathway in which coffee drinking seems to be an effect modifier (possibly through selection of mutated cells) rather than a direct aetiological agent. ${ }^{8}$ Porta et al did not find any signature as expressed by a characteristic mutational spectrum.

From the analysis I have suggested it is clear that in the history of medicine a variety of concepts of cause have been used, which fit different purposes. Current medicine is probably the most evident expression of the intertwining of different traditions. On the one hand, we have a rigorously empirical concept of proof, the one that dominates in the clinical trials on the efficacy of drugs: in this case the "gold standard" is the randomised trial, which is a strictly experimental approach based on the empirical comparison among different groups of patients with a heavy use of the probability theory. Attention is drawn towards a single cause because of the eminently practical (curative) goal. Epidemiology itself is largely dependent on probabilistic 
relations, but it does not refer any longer to single causes (not to speak of necessary causes), and in this sense it is related to historical "conditionalism". On the other hand, many types of research in physiology and physiopathology are based on a counterfactual experimental logic, and apply a conception of cause not very different from the one proposed by Mackie. Finally, molecular research almost literally refers to Salmon's idea of a mark that can be followed along time.

Rather than facing incompatible models of causality, I suspect we are simply considering different levels of reality. This is the context in which the new causal pathways suggested by the intertwining between the probabilistic science of epidemiology and the idea of "marks propagating over time" of molecular biology can create and reinforce new causal hypotheses.
University of Torino and CPO-Piemonte, Torino, Italy

1 von Engelhardt D. Causality and conditionality in medicine around 1900 In: Delkeskamp-Hayes C, Gardell Cutter MA, eds. Science, technology, and the art of medicine. Dordecht: Kluwer, 1993.

2 Mackie JL. Causes and conditions. American Philosophical Quarterly 1965;2: 245-52.

3 Hempel CG. Philosophy of Natural Science. Englewood Cliffs, NJ: Prentice-Hall, 1966.

4 Nuland SB. Doctors. The biography of medicine. New York: Vintage Books, 1988

5 Salmon W. Scientific explanation and the causal structure of the world. Princeton, NJ: Princeton University Press, 1984.

6 Vineis P, Malats N, Porta M, et al. Human cancer, carcinogenic exposures and mutation spectra. Mutat Res 1999;436:185-94.

7 Porta M, Malats N, Guarner L, et al. Association between coffee drinking and K-ras mutations in exocrine pancreatic cancer. PANKRAS II Study Group. F Epidemiol Community Health 1999;53:702-9.

8 Vineis P. ras Mutations and a cup of coffee: cause, confounder, effect modifier, or what else? F Epidemiol Community Health 1999;53:685. 